\title{
VIRTUAL LABORATORY AS REALISTIC TOOL FOR THE E-LEARNING IN THE ELECTRIC AND ELECTRONIC MEASUREMENT AND INSTRUMENTATION
}

\author{
Domenico Grimaldi ${ }^{1)}$, Sergio Rapuano ${ }^{2)}$, Mariella Riccio ${ }^{2,3)}$, Francesco Zoino ${ }^{2,3)}$ \\ ${ }^{1)}$ DEIS, University of Calabria, 87036 Rende (CS), E-mail: grimaldi@deis.unical.it, \\ 2) Dept. of Engineering, University of Sannio, Corso Garibaldi 107, 82100 Benevento, E-mail: rapuano@unisannio.it, \\ ${ }^{3)}$ Didagroup S.p.A., Viale dei Sanniti, 1, S. Giorgio del Sannio (BN), E-mail: \{mriccio, zoino\}@didagroup.it.
}

ITALY

\begin{abstract}
The paper deals with the research project adopting the e-learning methodologies for teaching in electrical and electronic measurement and instrumentation. The objective is to offer to the users the tool to achieve accurate and practical experience by working in real conditions and on real instruments. After the description of both the hardware and the software architecture of the Remote Didactic Laboratory, Laboratorio Didattico Remoto - LA.DI.RE. "G. Savastano", the innovative solutions addressing the requirements of reality are examined and investigated. The core of the system is the Learning Management System (LMS) that provides didactic contents, enabling the account management, security protection, collaborative learning, student activity tracking, and feedback collection. LA.DI.RE. adds to the commercial LMS the innovative services dedicated to laboratory activities concerning the remote control of real instrumentation and the real-time visualization of instrument front panels during the execution of the experiment chosen by the user.
\end{abstract}

Keywords: e-Learning, virtual laboratory, education, distributed measurement systems.

\section{INTRODUCTION}

Talking about e-Learning means, most of all, talking about both of methodology and technology of the Internet-based distance learning paradigm.

The term e-Learning is known since a lot of time in both fields of life long learning and in academic research as determined, at first, by the Sorbonne declaration of May 25, 1998, that emphasized the creation of the European area of higher education as a key way to promote citizens' mobility and employability and the Continent's overall development [1].

The declaration of Bologna in June 19, 1999 aimed to create an European Higher Education Area by 2010 , in which students could choose from a wide and transparent range of high quality courses and benefit from smooth recognition procedures. Moreover, the e-learning aimed to standardize the European higher education while respecting the fundamental principles of autonomy and diversity [2]. At least it follows the Lisbon Strategy that is heavily based on innovation as the motor for economic improvement, the "learning economy" and at the end the social and environmental renewal to empower European economic and social development [3].

The Italian University system, currently attends, from one side, to the confirmation of many academic eLearning centre, from the other side to the confirmation of development of distance universities that highlights the importance of the research path directed to empower the declared capabilities of the e-Learning methodologies.

Currently the state of art of the software systems for eLearning is represented by environments specifically designed for the management of the learning objects and of the user learning path. This environment is named Learning Management System (LMS).

From the point of view of the educational content management, the LMS offers many functionalities that allow organizing the contents as standardized Learning Objects (LOs), structured inside the learning path and well designed in accordance with the learners' needs.

Moreover, the LMS facilitates the interactions and the collaboration among the users due to specific collaborative environments, both synchronous and asynchronous. From the point of view of the teachers, the LMS offers a set of functionalities to follow the evolution of the learning activities of their students and to evaluate their skills.

The paper deals with a research project adopting 
e-Learning methodologies in the field of electronic engineering education. The project resulted in the realization of an innovative distance learning environment including the typical features of commercial LMS and the capability of managing and remotely controlling electronic measurement instrumentation to give, to the students, the possibility of executing experimental activities on actual instrumentation in realistic conditions.

This innovative system, called Remote Didactic Laboratory Distributed over a Wide Area Network (Laboratorio Didattico Remoto - LA.DI.RE. "G. Savastano"), simplifies the most relevant step in the learning process, especially in scientific and technological fields, that is the application of the acquired theoretical knowledge. In fact, practical training on actual instrumentation is essential in order to assure a good knowledge transfer from teacher to students and to train good professionals. However, electric and electronic measurement laboratories, both public and private ones, are not widespread, due mainly to their total cost of ownership, and this makes difficult the life-long learning of specialized technicians, especially in the field of process control, quality control and test engineering.

The LA.DI.RE. "G. Savastano" overcomes such limits by sharing the limited resources of a network of remotely accessible laboratories among several teachers and students located in different countries. It provides the people involved in all kinds of educational activities with the instruments for: (i) following or doing interactive web lessons and labs; (ii) learning by doing on actual instruments; (iii) making common projects; (iv) exchanging comments and experiences; (v) doing examinations; (vi) tracking the student activities and monitoring their degree of knowledge [4].

The user access is granted by means of Internet and can be done at any hour of the day. There aren't particular software requirements, indeed the download of large software packages is not needed.

From the experiment developer and maintainer point of view, any kind of software controlling electronic instrumentation can be included in the system and can be made remotely accessible without rewriting anything of the original application.

In this paper the authors will thoroughly analyze the LA.DI.RE. "G. Savastano" Virtual Laboratory (VL), from two points of view. First they will discuss the learning environment form the technical and didactical point of view highlighting most of the innovations introduced like the multi-channel evolution, On the other hand they will discuss the international diffusion of the VL and the services and facilities specifically created for the enterprise.

\section{E-LEARNING AND ACTUAL LABORATORIES}

The VLs proposed in literature to perform didactic activities in the field of electronic engineering are mainly concerned with a common target: to allow the execution of experiments involving actual instrumentation and circuits under test, rather than simulating them, even in virtual reality environments.

The advantages of VLs are: (i) they can share the limited resource of the laboratory among several teachers and students, addressing some problematic issues coming from the management of laboratories for a lot of students, (ii) they are available seven days a week at any time of the day, and (iii) they are very cost-effective.

The proposed hardware infrastructures are similar, they have a modular architecture and contain multiple reconfigurable sub-systems, hardware, software or both.

For example, the laboratory proposed in [4] is versatile, in terms of hardware resources, due to the dynamic reconfiguration capability and the low cost of the hardware components. Moreover, this system enables the users to test their own custom circuit designs.

Concerning the software architecture of the VL a set of recommendations can be found in the literature. The main targets are: flexibility, modularity, generality and hardware independence [5], [6].

The software architectures proposed in [5], [6] have a layered structure that makes them suitable for implementation of versatile measurement systems for didactic aims. The main difference between [5] and [6] is the approach to the control of instrumentation. In [6] the measurement functionality is encapsulated in a hardwareindependent layer that communicates with its environment, e.g. physical hardware, through intermediary software components. In [5], the measurement software subsystem is hardwaredependent, while the access and communication subsystems are platform independent, so that any application controlling some kind of instrumentation can be remotely controlled. The first approach is mainly oriented to the easy development of new Virtual Instruments (VIs), the second one address also the reusability of the existing VIs, independently from the language used for developing them.

One of the most relevant problems designing the laboratory subsystem is the remote access to the 
experiment. The main design objectives taken into account in order to provide the remote access to the VIs were the following:

(i) portability: the visualization environment has to be portable on different hardware and operating systems;

(ii) usability and accessibility: the visualization and the management of an experiment have to be easy to understand and to perform, even for users that are not expert of information technologies, and the system features have to be accessed easily by students operating at University laboratories or at home;

(iii) maintenance: the maintenance costs should be reduced. This can be made possible through a client-server approach that eliminates the need for installing and upgrading application code and data on client computers;

(iv) client-side common technologies: students have to access to the system using their desktop computers based, with no need of powerful processors or high memory capacity, and connecting to Internet through low speed dialup connections;

(v) security: the remote access of the students through the Internet must preserve the integrity of recorded and transmitted data and of the system as a whole;

(vi) scalability: the system performance has not to be affected when connected users increase.

Most of the proposed VLs cannot be considered an effective platform for delivering distance education. From the technical point of view the distance learning paradigm is based on LMS and Content Learning Management Systems (CLMS) which are based on the client-server application model to deliver both administrative services and didactical synchronous and asynchronous facilities for the learning process.

The administrative services allow to manage learners and to allocate learning resources such as registration, classroom, instructor availability, instructional material, fulfillment and on line delivery.

The CLMS usually includes an LMS and adds an authoring system providing an infrastructure that can be used to rapidly create, modify, and manage content for a wide range of learning. The CLMS can retrieve detailed data on learner scores, question choices, navigation habits and use them to give to the content managers crucial information on the effectiveness of the content when combined with specific instructional strategies, delivery technologies, and learner preferences.

The synchronous services allow learners to perform real time interactions among themselves or among learners and their teacher, to visualize the speaker and to share the speaker's desktop during a lecture. The asynchronous services, on the other hand, are represented by forum or classroom email. They don't include real time interactions with the teachers.

The LA.DI.RE. "G. Savastano" VL [4] is an innovative distance education system, including the VL for the experimental activities, oriented to teaching of electric and electronic measurement subjects.

The fusion of the innovative VL and the commercial LMS, provides a set of didactical and administrative tools. Most of the LMS allows tracking the user's progress and performance across all types of training activities. Moreover, the use of the standard LMS ensures the interoperability and portability of the LOs.

The next innovative aspect of the proposed environment is the distributed architecture that involves different physical laboratories which are geographically distributed at national and international level [7].

This distance learning environment, moreover, allows to empower the feeling of working on real instruments and in real conditions using some technological solutions based on ip-camera to visualize in real time the evolution of the experiments [8], [9].

LA.DI.RE. "G. Savastano" is the first proposal that avoids specific client-side applications and uses the thin-client approach to transfer the processing of the software to the server [10].

\section{EDUCATION ON INSTRUMENTATION AND MEASUREMENT}

The LA.DI.RE. "G. Savastano" is able to provide the students of electric and electronic measurement courses with the access to remote measurement laboratories to deliver different didactic activities related to measurement experiments.

The main infrastructure is composed by the laboratories at the University of Sannio and at the University of Reggio Calabria "Mediterranea" under the patronage of the National Research Association on Electric and Electronic Measurement (GMEE) and the collaboration of about twenty Italian Universities and some specialized instrumentation, e-learning and publishing companies such as National Instruments, Tektronix, Agilent Technologies, Yokogawa, Keithley, Rockwell Automation, Didagroup and Augusta publishing.

The core of the system is the LMS that is under the control of the administrator, and it interacts with a run-time environment which is addressed by learners, who in turn may be coached by a trainer. 
These three kinds of participant to the e-learning process can be logically and physically distributed, i.e., installed on distinct machines in different sites, and provided by different vendors or content suppliers. In order to make such a distribution feasible, standards such as the Learning Object Metadata (LOM) [11] specifications, the Aviation Industry Computer-Based Training Committee (AICC) [12] and the Instructional Management Systems (IMS) guidelines [13], the Sharable Content Object Reference Model (SCORM) [14], try to ensure: (i) plug-and-play compatibility, (ii) interoperability, (iii) accessibility, and (iv) reusability of Web-based learning content.

According to almost all the international guidelines, the educational contents are handled, stored, and exchanged in LOs. Basically, LOs are units of study, exercise, or practice that can be followed in a single session. They represent reusable modules that can be authored independently of the delivery medium and can be accessed dynamically, e.g. via the Web [15]. Ideally, LOs can be exchanged between different LMSs and plugged together to build classes that are intended to serve a particular purpose or goal.

LOs can be stored in a relational or an objectrelational database and are typically broken down into a collection of attributes, some of which are mandatory, and some of which are optional. In a similar way, other information relevant to a learning system (e.g., learner personal data, learner profiles, course maps, LO sequencing or presentation information, general user data, etc.) can be mapped to common database structures. These activities can be modelled as processes or workflows and can be attributed to and associated with the various components of a learning platform.

For this reason the idea is to use the potentiality of remote teaching [16] and, in particular, the use of the Internet as a channel to reach the students or workers at their homes was soon recognized [17]-[19]. As the offthe-shelf LMSs are usually closed, proprietary software systems, often not customizable at all, the research carried out by scientists to provide teaching of electric and electronic measurement including experiments didn't take in account the possibility of integrating remote laboratories and LMSs.

The LA.DI.RE. "G. Savastano" system instead integrates an off-the-shelf LMS and a geographically distributed laboratory. The next sections describe the main services provided focusing on the distributed laboratory accessed through the LMS. In particular it will be described the distributed system, its architecture and innovative functions as well as its integration with the LMS (Fig.1).

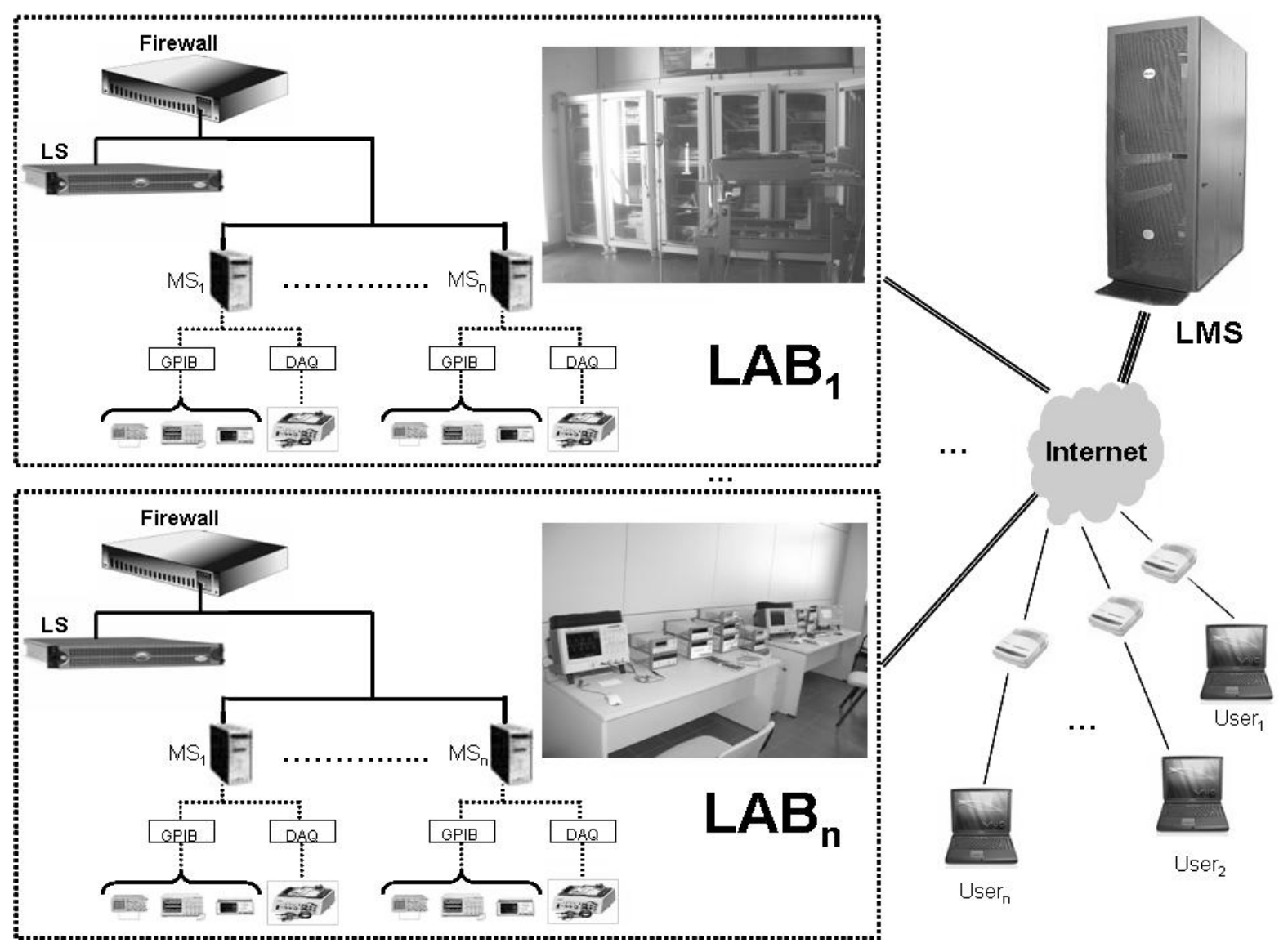

Fig. 1 - Hardware components of the proposed architecture. 


\section{REMOTE CONTROL OF ACTUAL INSTRUMENTATION}

In order to allow the student to access the remote and geographically distributed didactic laboratory, the Web-based multi-tier distributed architecture centered on the LMS is adopted. This can be considered as the core component of the overall system [20].

The paradigm that consents the user to access to the system without specific software on client side is the thin-client. It is a distributed paradigm in which the network separates the presentation of the user interface from the application logic. It is a clientserver architecture in which the application execution and data management is performed on the server, called Terminal Server. This technology has been chosen after a comparison with other technologies taking in account some specific reference parameters as: (i) the reusability of the existing VIs, (ii) the rapid development of new VIs, (iii) the Plug-in dimension, (iv) the portability, (v) the remote visualization, (vi) the remote control and (vii) the remote design. The comparison, given in Tab.1, shows that the thin client technology assures the best performances concerning the considered reference parameters. By using thin client technologies students are also able to use limited hardware devices, the so called thin-client devices equipped only with a processor and a flash memory (no hard disk or other storage units) such as Personal Data Assistant (PDAs), handheld devices, mobile phones. This solution extends the class of possible learners to mobile users, owning a smart phone, a PDA or a notebook with a modem or a wireless LAN adapter.

Table 1. Technology comparison

\begin{tabular}{|l|c|c|c|c|c|c|c|}
\hline & $\begin{array}{c}\text { Lab.Rem. } \\
\text { Panels }\end{array}$ & Datasocket & AppletView & CORBA & RMI & $\begin{array}{c}\text { XML. } \\
\text { SOAP }\end{array}$ & $\begin{array}{c}\text { Thin. } \\
\text { client }\end{array}$ \\
\hline $\begin{array}{l}\text { Reusability of } \\
\text { existing Virtual } \\
\text { Instruments }\end{array}$ & High & $\begin{array}{c}\text { Low } \\
\text { (GUI dev) }\end{array}$ & High & $\begin{array}{c}\text { Low } \\
\text { (GUI } \\
\text { dev) }\end{array}$ & $\begin{array}{c}\text { Limited } \\
\text { to JAVA }\end{array}$ & $\begin{array}{c}\text { Low } \\
\text { (GUI } \\
\text { dev) }\end{array}$ & High \\
\hline $\begin{array}{l}\text { Rapid development of } \\
\text { new Vls }\end{array}$ & Yes & No & Yes & No & No & No & Yes \\
\hline Plug-In dimension & High & High & $\begin{array}{c}\text { Low } \\
\text { (JVM) }\end{array}$ & $\begin{array}{c}\text { Low } \\
\text { (JVM) }\end{array}$ & $\begin{array}{c}\text { Low } \\
\text { (JVM) }\end{array}$ & $\begin{array}{c}\text { Low } \\
\text { (JVM) }\end{array}$ & $\begin{array}{c}\text { Low } \\
\text { (JVM) }\end{array}$ \\
\hline Portability & $\begin{array}{c}\text { Limited to } \\
\text { So supp. } \\
\text { by NI }\end{array}$ & $\begin{array}{c}\text { Limited to } \\
\text { so supp. } \\
\text { by NI }\end{array}$ & High & High & High & High & High \\
\hline Remote Visualization & Yes & Yes & Yes & Yes & Yes & Yes & Yes \\
\hline Remote Control & Yes & Yes & Yes & Yes & Yes & Yes & Yes \\
\hline Remote Design & No & No & No & Limited & Limited & No & Yes \\
\hline
\end{tabular}

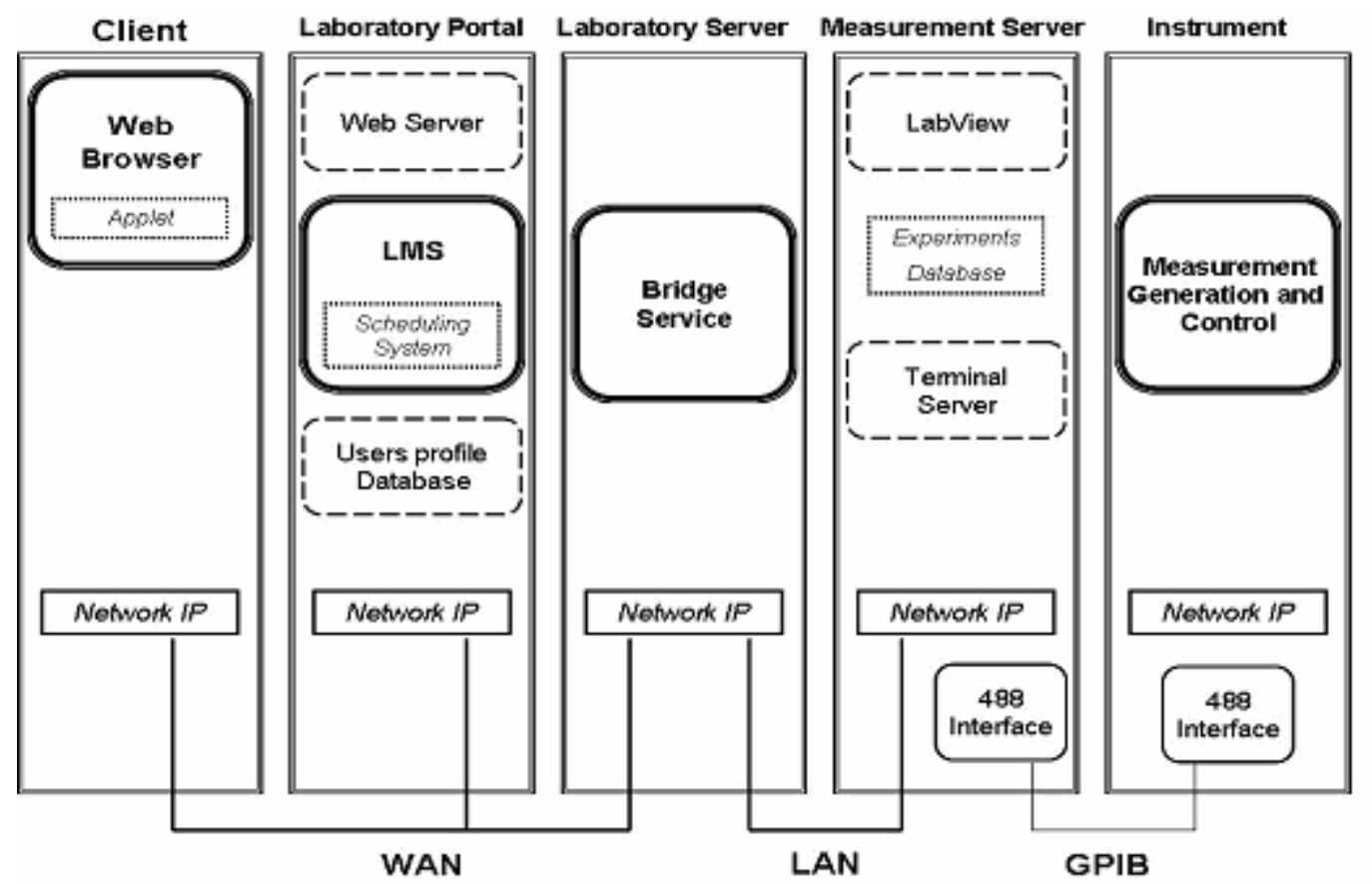

Fig. 2 - Software layers of the components of the laboratory architecture. 
The thin-client paradigm has been adopted by designing a multi-tier architecture, shown in Fig.2, composed of the following three tiers:

i. the presentation-tier: it manages the experiment visualization on client-side. It is based on standard Web browsers. The only software component needed is the Java 2 Runtime Environment, which is used in order to employ the Java Applet technology for the experiment access and control from the client machine.

ii. the middle-tier: it manages the system logic on the server-side. It includes the following components:

- LMS, executed on a central server of the overall e-learning environment, called Laboratory Portal. The LMS interfaces to the users through a Web Server, that is hosted on the same machine.

- Laboratory Server (LS) that is used to interface the laboratory with the rest of the distributed architecture. There are LS for each measurement laboratory of the universities involved in the project. It delivers the access and the control to the measurement equipment through a service, called Bridge Service. Moreover, the LS is the only machine in a laboratory directly accessible from the Internet, while the other servers are connected in a private local network. For this reason the LS can also be used for security purposes in order to monitor the accesses to the measurement laboratory and to protect it against malicious accesses.

- Measurement Server (MS) that is a PC enabling the interaction with one or more instruments. The MS is physically connected to a set of programmable instruments through an interface card.

The used VIs, that are the software modules to control the real instrumentation developed in LabVIEW environment, are stored in a database of the MS, where the LabVIEW environment is installed. No adjustment is necessary to include the VI in the virtual learning environment. Therefore the wide number of existing VIs can be reused without requiring additive work.

iii. the storage-tier: it performs the data management related for example to the user profiles and the distributed management of the data related to the available experiments at the different measurement laboratories. It is based on a series of geographically distributed databases, managed by using the Relational Database Management System.

The Web and Java technologies have been adopted to implement the described architecture, in order to allow a student, using exclusively a common Web browser with a Java Virtual Machine, the remote visualization and control of an experiment, ensuring a high system portability and usability. The student obtains on his/her computer the displaying of the application executed on the MS and used to control the instrumentation for the requested experiment.

\section{INNOVATIVE CHARACTERISTICS OF LA.DI.RE. "G. SAVASTANO"}

The remote laboratory is distributed on geographical scale since the laboratory resources (multimedia contents and real instrumentation) are located physically in laboratories belonging to different universities. At the moment four laboratories are involved. Two of them have been realized and operate, at the University of Sannio in Benevento, and at the "Mediterranea" University of Reggio Calabria in Italy. Two more laboratories are going to be realized at the University of Zagreb (Croatia) and at the Technical University of Kosice (Slovak Republic). The access requests to the measurement instruments are handled by a distributed scheduling system that verifies the catalogue of available measurement instrumentation, and re-directs the user request to the measurement laboratory, chosen among the partner laboratories, in which the required instrument is currently available.

The scheduling is based on a distributed database that contains the set of all the resources belonging to each physical laboratory and their mapping scheme in the system. The scheduling flow chart is reported in Fig.3. After the access request of the client the LMS verifies the availability of the desired experiment through specific queries to the LSs that manage such kind of resources. The scheduler selects the LS where the resources chosen are available, assigns the resources to the client and sets their status as busy. Then the LMS sends to LS the necessary information to set up a direct link with the requesting client. The LS starts the connection and routes the data stream concerning the experiment between the client and MS. After 1 hour the connection will be closed, the client will be informed about this with a message and the next user waiting for the experiment will be informed that he/she can access it. Of course, the time out can be modified depending on the specific experiment. If the access request cannot be satisfied as all the resources are busy the LMS informs the client of this temporary situation.

The developed architecture has to assure a very high security. For this reason the only external interface to Internet is Linux-based firewall that manages the data transmitted between two Ethernet ports, one is connected to the external network and the other one is connected to the LAN. The firewall applies a Network Address Translation (NAT) in order to mask the IP addresses of the servers that should access Internet. Concerning the VL realized at the University of Sannio, the LMS and the LS are in the same LAN. Therefore 
the LMS and LS only are accessible via Internet. Every communication with the MSs is possible through the LS thanks to the previously introduced bridge service.

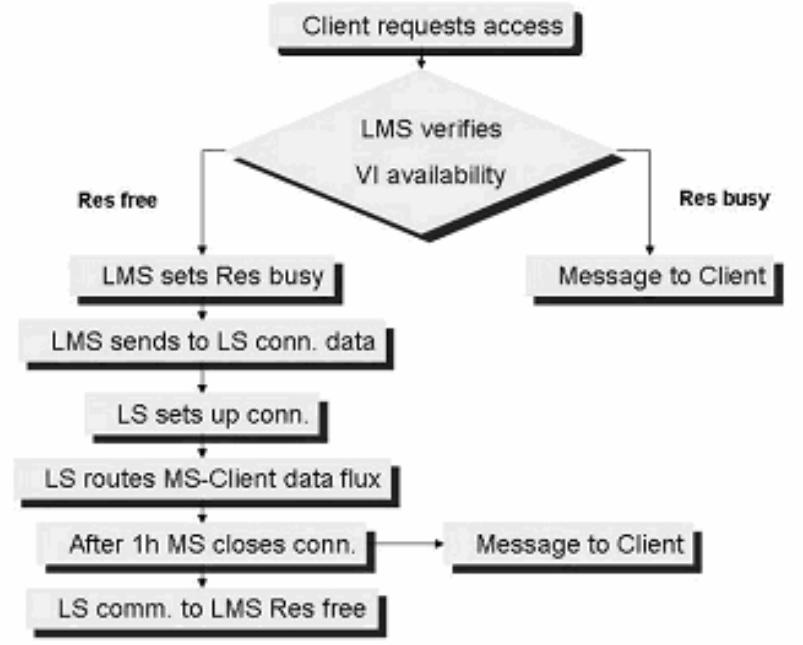

Fig. 3 - Scheduling flowchart.

The LA.DI.RE. "G. Savastano" system presents some classical services that allow to the user to browse in the e-Learning environment developed to access to the didactic contents. Every user has a specific home page with the courses assigned and other functionalities as university personal booklet, personal profile, historical booklet and so on. In each course is possible to use some specific community activity as the forum, the chat, the mail, the "bacheca" functionality.

The complete access procedure to LA.DI.RE. "G.Savastano" system is composed by some specific phases:

1. connection to the link www.misureremote.unisannio.it (Fig.4);

2. authentication phase;

3. choice of the course;

4. choice of the didactic content;

5. setting of some parameters concerning the operative system version and the video resolution;

6. execution of the experimental content and the visualization of the front panels of the instrumentation involved in the selected experiment, thanks to specific video capturing devices (Fig.5).

A very important service is the real-time visualization of the front panels of the instrumentation during the execution of the experiment. The LA.DI.RE. "G. Savastano" includes a system dedicated to management of video devices to allow to the student to observe the effects of his actions on the instrumentation involved in the experiment. A method to manage the video streaming data coming from different codecs has been proposed in [21]. Within that work it has been proposed to acquire a video from each instrument of the measurement laboratory by means of a video acquisition device connected to the MS. A video bridge component is responsible for sending the instrumentation video received from the MSs to the requiring user. The proposed approach relies on the Real Time Protocol (RTP) instead of the RDP used for remote access to the VIs. In fact, RDP is neither effective nor flexible for the video transmission since it doesn't permit to modify parameters such as codec, video formats, frame rate and involves a very high bandwidth occupation.

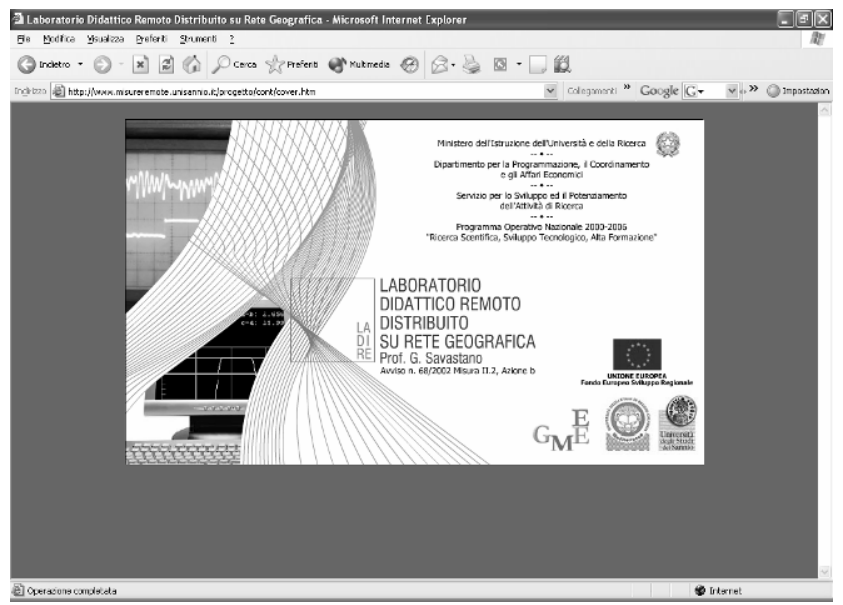

Fig. 4 - Web portal of LA.DI.RE. "G. Savastano".

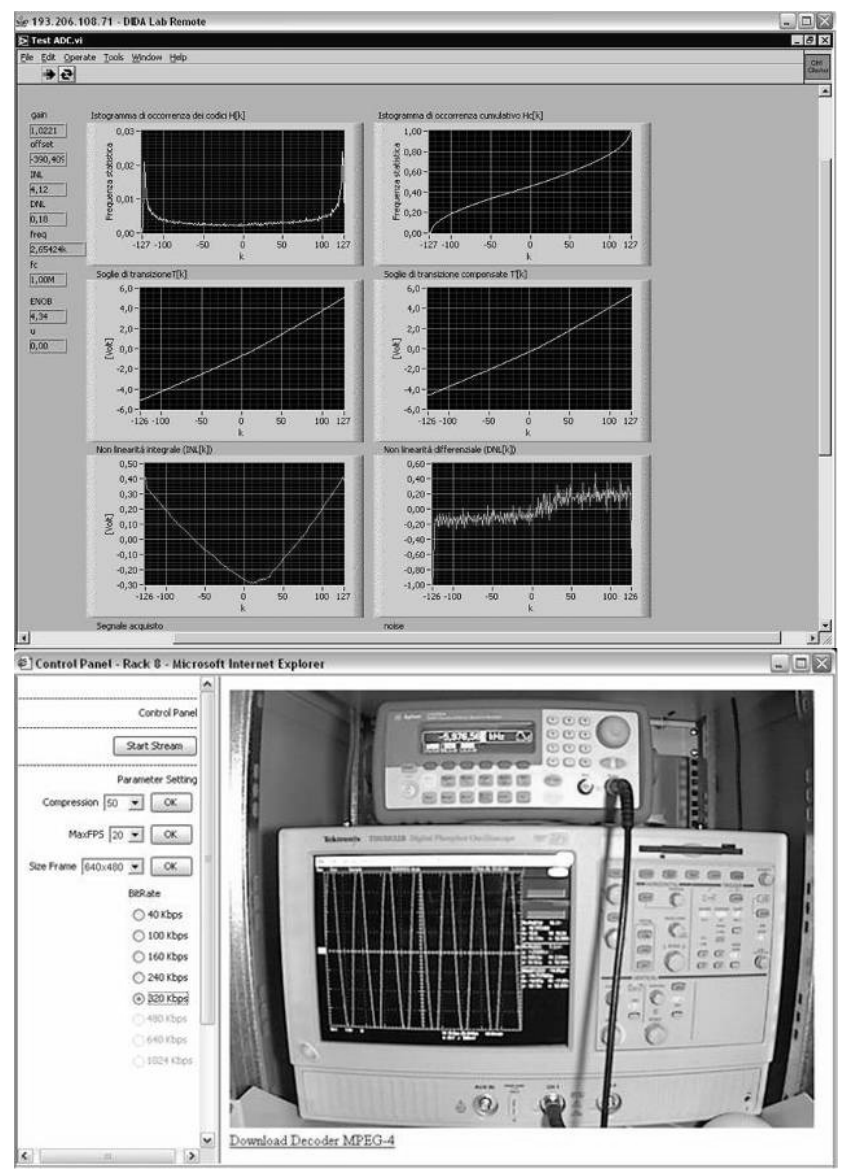

Fig. 5 - Execution of the experiment and visualization of the used instruments. 
The software architecture is formed by a server and a client components developed in Java that enable the delivering of the instrument videos. For acquiring the videos an Axis 207 IP camera with an integrated MPEG-4 encoder has been used [21].

The LS starts a direct communication with the client delivering both the instrumentation access through the VI front panel and the video stream transmission coming from the IP camera associated to the chosen experiment. In this case the video stream has an independent part from the MS. No application runs on the MS. Before starting the experiment, the student can choose among different displaying options such as no visualization, photo displaying with different resolutions, visualization of one frame per second with different resolutions, visualization of 10 frames per second with different resolutions and visualization of 25 frames per second with different resolutions. Only the options compatible with the available bandwidth estimated by the system will be enabled on screen, as described in [21].

This specific service consents to provide other innovative services like the remote demo, that is very important for the producers of measurement instruments (Fig.6). In this way the purchasers can test the characteristics of the new instrument connecting to LA.DI.RE. "G. Savastano" and so observe the front panels of the specific instrument by remote thanks to the real-time visualization service.

Three different user profiles are managed by the system: student, teacher and administrator.

The student can access to the system after the authentication phase and access to courses presented in his home page. In particular the services delivered by the remote measurement laboratory module to the student are mainly the following: (i) Synchronous virtual laboratory, this service allows the student to follow on-line a laboratory activity hold by the teacher. The student can see on his/her display the desktop of the server used by the teacher to control the measurement instruments involved in the experiment. The experiment is carried out by operating on the front panel of the LabVIEW VI controlling all the involved instrumentation. Of course, the students should be logged in the system during the scheduled lab session. The data stream from the physical laboratory to the students can be sent in multicast mode. No automated scheduling policy is foreseen for such kind of activity.

(ii) Experiment visualization, this service allows the student to observe the automatic execution of the experiment to take practice with the experiment procedure (Fig.7). This kind of service can de delivered to the students at each time of the day and all the times they need it without supervision. Therefore it is an asynchronous service.

(iii) Experiment Control, this service allows the student to perform an experiment controlling remotely one or more instruments and observing them by means of a camera. The student can choose a specific experiment in a set of predefined ones and he can run it only if the required measurement instruments are currently available (Fig. 8).

(iv) Experiment Creation, this service allows the student to create remotely an experiment interacting directly with specialized software executed on the servers used to control the measurement instruments. This feature enables the adoption of PBL as didactic model. Under the supervision of the teacher, the students can develop a specific project producing, in an individual or collaborative manner, a VI to control a set of real instruments (Fig. 9).

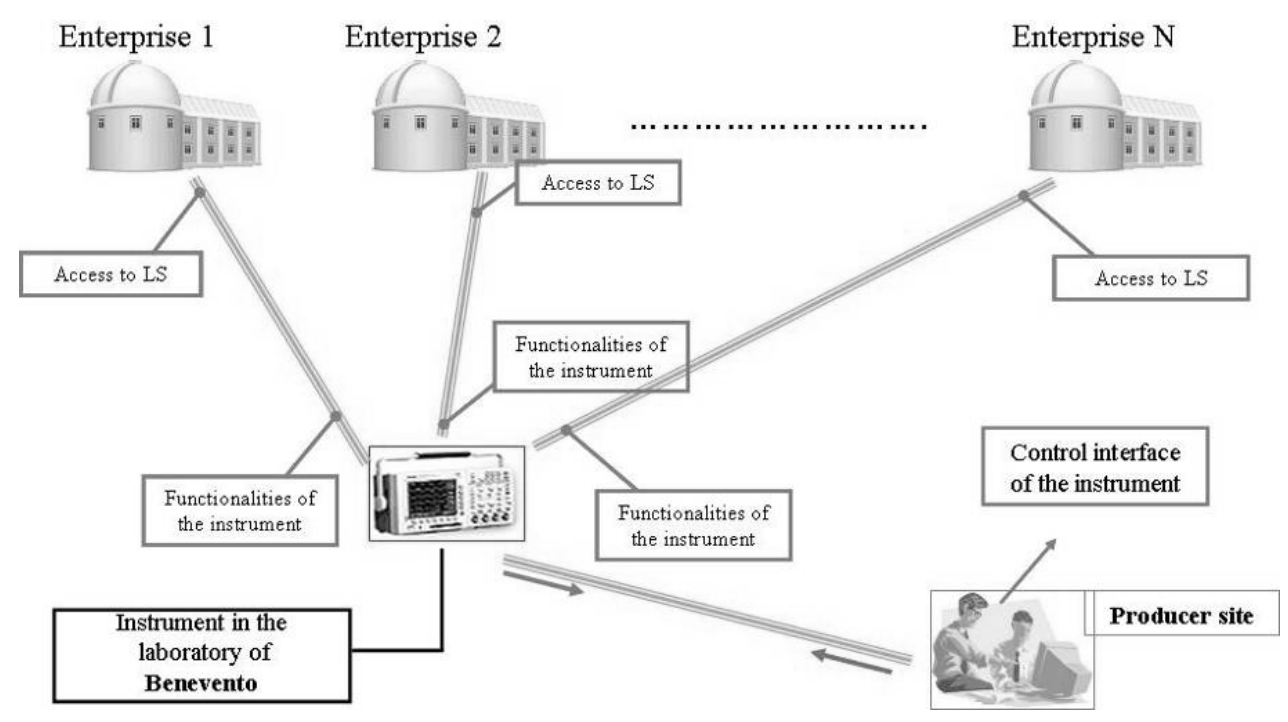

Fig. 6 - Remote demo service of a new instrument. 


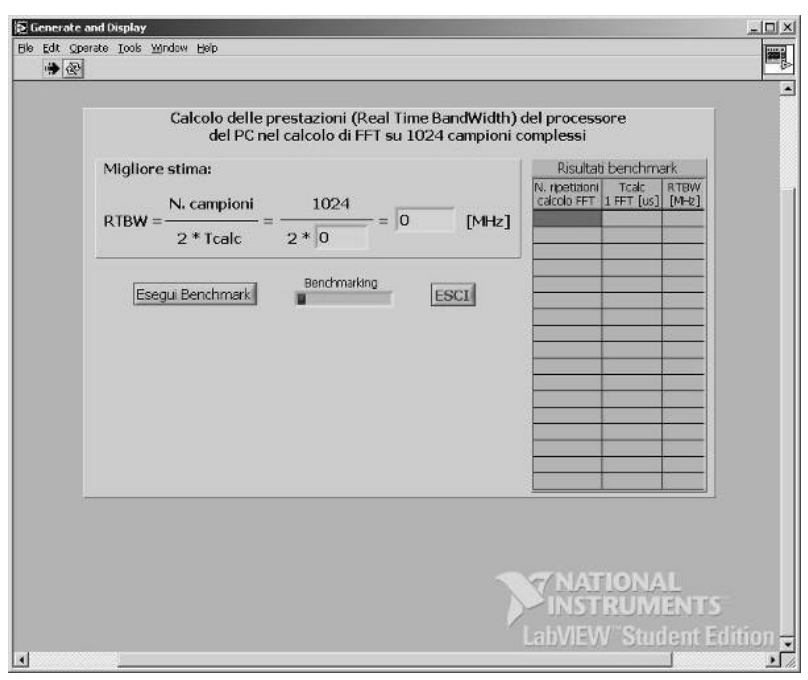

Fig. 7 - Synchronous virtual lab/experiment visualization.

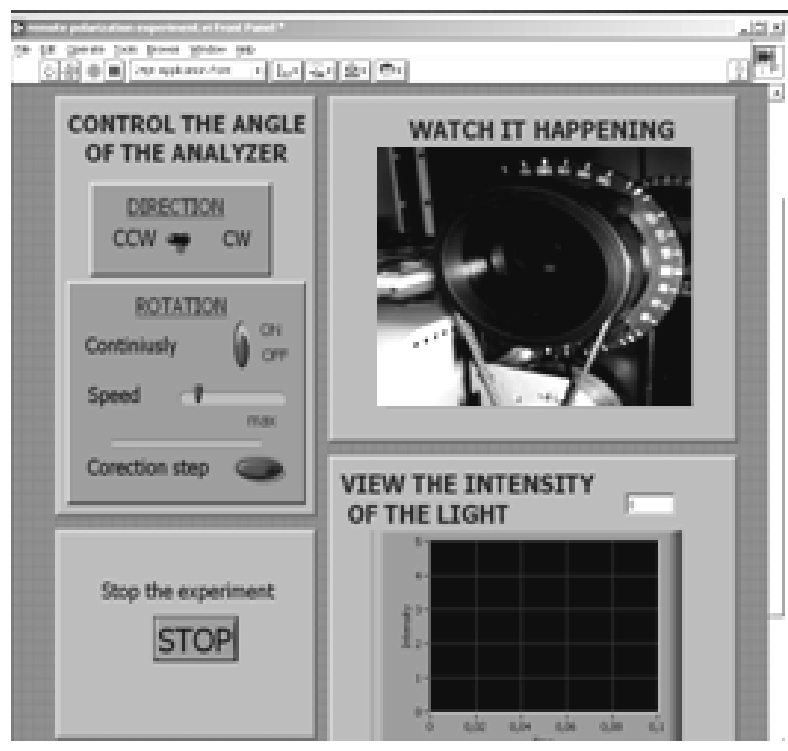

Fig. 8 - Experiment control.

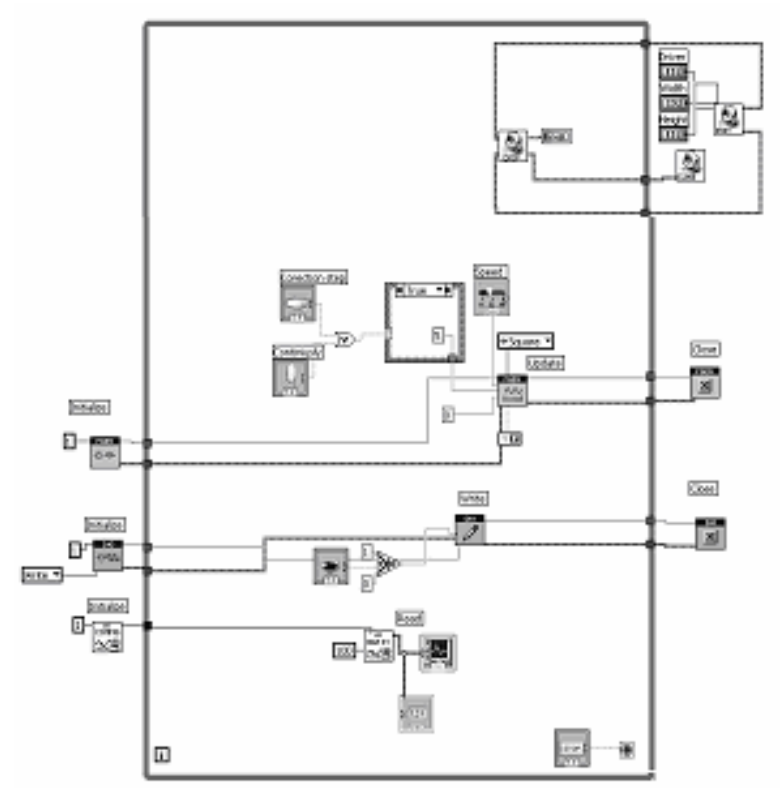

Fig. 9 - Experiment creation.
The services (ii), (iii) and (iv) require a unicast data transmission for each experiment and lock a laboratory resource for the time required by the students to carry out all the experimental activities. Therefore the scheduling policy is necessary to grant transparently the access to a specified resource.

The services delivered to a teacher are related to the remote handling of the available experiments: remote creation, updating and removal of experiments. Currently the experiments are created as VIs realized by the teacher or the tutor. The set up of an experiment is the same as it would carry out locally. The instruments should be connected to the circuit or device under test and the VI has to be only copied in a specific directory in the MS. Also if the hardware set up has to be carried out in the laboratory, the VI can be produced in the teacher working place and then transferred to the MS by means of the above quoted services.

Finally the administrator is responsible of the correct working of the overall distributed system and of handling the user profiles. He manages the communication with the teacher to solve the possible problems concerning the platform and can change the profile characteristics, assigning different functionalities to the user.

\section{CONCLUSIONS AND FUTURE WORKS}

The paper presented a distance learning system for teaching in electric and electronic measurement and instrumentation. The core of the system is the standard Learning Management System (LMS) that provides didactic contents, enabling (i) the account management; (ii) the security protection; (iii) the collaborative learning; (iv) the student activity tracking, and (v) the feedback collection.

The experiments are managed by the students from their homes requiring a web browser only. The system adds to the classical services of a commercial LMS other innovative services dedicated to laboratory activities concerning the remote control of real instrumentation and the real-time visualization of instruments front panels during the execution of the experiment chosen by the user.

The ongoing activity regards the extension of the system access to mobile users and to make a test on the effectiveness of the didactics.

Another important future work will be the migration of the same architecture on broadcast system by the Digital Terrestrial Television that will assure the extension of the learning services to a more wide set of citizens. 


\section{REFERENCES}

[1] http://cordis.europa.eu/itt/itt-en/05-1/policy01.htm

[2] http://cordis.europa.eu/search/index.cfm?fuseaction $=$ news. simpledocumentLucene $\& \mathrm{RCN}=13695 \& \mathrm{CF}$ $\mathrm{ID}=1859411 \&$ CFTOKEN $=86500$

[3] http://www.crue.org/sorbo-in.htm

[4] K. Efstathiou, D. Karadimas and K. Zafeiropoulos, "A Remote Electrical Engineering Laboratory based on Re Configurable Hardware" Proc. of IEEE IMTC, Warsaw, Poland, 2007.

[5] S. Rapuano, F. Zoino, "A learning management system including laboratory experiments on measurement instrumentation", IEEE Trans. on Instrumentation and Measurement, vol.55, N.5,2005, pp.1757-1766.

[6] D. Wisell, P. Stenvard, A. Hansebacke, N. Keskitalo, "Considerations when Designing and Using Virtual Instruments as Building Blocks in Flexible Measurement System Solutions " Proc. of IEEE IMTC, Warsaw, Poland, 2007.

[7] N. Ranaldo, S. Rapuano, M. Riccio, E. Zimeo, “A Platform for Distance Learning including Laboratory Experiments", Proc. of "Congresso Annuale AICA 2004, Ricerca ed Impresa: Conoscenza e Produzione per la Società dell'Informazione", Benevento, Italia, 2004, pp. 503-516.

[8] N. Ranaldo, S. Rapuano, M. Riccio, F. Zoino, “A Remote Laboratory for Electric Measurement Experiments: The Remote Displaying of Instruments", Proc. of "The 19th Metrology Symposium”, Abbazia, Croazia, 26-28 Sept. 2005.

[9] N. Ranaldo, S. Rapuano, M. Riccio, F. Zoino "On the use of video-streaming technologies for remote monitoring of instrumentation", Proc. of IMTC, Sorrento, Italy, 2006 pp.861-867.

[10] N. Ranaldo, S. Rapuano, M. Riccio, E. Zimeo, “A Thin-Client Approach for Distance Learning of Measurement Instrumentation", Proceedings of "The 4th IASTED International Conference on Web-based Education", ACTA Press, pp. 649-654, Grindelwald, Svizzera, 21-23 Feb.2005.

[11]IEEE Standards Department, "IEEE Standard 1484.12.1 for Learning Object Metadata", IEEE Publication, 2002.

[12]Aviation Industry CBT Committee Guidelines and Recommendations. www.aicc.org.

[13] Instructional Management Systems Global Learning Consortium, "IMS content packaging specification 1.1.4", 2004. www.imsglobal.org.

[14]Advanced Distributed Learning, "Sharable content object reference model, 2nd Edition", 2004. www.adlnet.org.
[15]C. P. Chu, C. P. Chang, C. W. Yeh, Y. F. Yeh, "A web-service oriented framework for building SCORM compatible learning management systems", Proc. of the Int. Conf. on Information Technology: Coding and Computing (ITCC'04), Las Vegas, Nevada, USA, vol.1, 2004, pp.156-161.

[16]Laboratory of Signal Processing and Measurement Information - Engineering Faculty of University of Sannio. http://lesim1.ing.unisannio.it.

[17]Agilent Technologies Educator's Corner. http://www.educatorscorner.com/experiments/inde $\mathrm{x} . \mathrm{shtml}$

[18]V.M.R. Penarrocha, M.F. Bataller, "Virtual instrumentation: first step towards a virtual laboratory", Proc. of the IEEE Int. Workshop on Virtual and Intelligent Measurement Systems, Annapolis, Maryland (MD), USA, 2000, pp.52- 56.

[19]A. Bagnasco, A.M. Scapolla, "A grid of remote laboratory for teaching electronics", $2^{\text {nd }}$ Int. Learning Grid of Excellence WG Workshop on eLearning and Grid Techn.: a fundamental challenge for Europe, Paris, France, 2003.

[20]J. Nieh, S.J. Yang, and N. Novik, "Measuring ThinClient Performance Using Slow-Motion Benchmarking", ACM Trans. on Computer Systems, vol.21, No.1, 2003 pp.87-115.

[21]N. Ranaldo, S. Rapuano, M. Riccio, F. Zoino, "Remote control and video capturing of electronic instrumentation for distance learning ", IEEE Trans. on Instrumentation and Measurement, vol.56, N.4, 2006, pp.1-10.

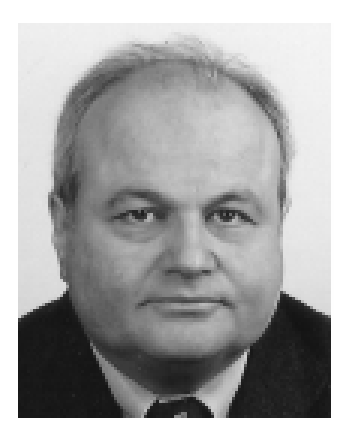

Domenico Grimaldi received the Dr. Ing. degree "cum laude" in Electrical Engineering from the University of Naples in 1979. He, after working as an independent consultant, in the 1990 joined the Department of Electronic, Computers and System Science (DEIS) of the University of Calabria, Italy, where he is currently Associated Professor of Electronic Measurement. He has remained there in a variety of research and management positions. From 1999 to 2006 he was responsible of the research unit in the frame of National Project PRIN supported by the Italian Ministry for University and Research. From 1997 to 2001 he was responsible in the Tempus Project and Leonardo da Vinci Project supported by the European Union. From 1998 to 2004 he was delegate of the Rector of the University of Calabria. He is a member of the Italian Institute of Electrical Engineers (AEI), and from 1998 to 2000 he was AEl vice-president for the Calabria Region. Since 1994 he is a member of the IEEE. He is coordinator of the Working Group "e-tools for I\&M Education" of the IEEE Instrumentation and 
Measurement Society, Technical Committee TC-23 "Education in Instrumentation and Measurement". Prof. Grimaldi has authored and co-authored over 160 papers published in international journals and conference proceedings. His current researches include the characterization of measurement transducers, neural modelling for $A D C$ and measuring systems, digital signal processing for monitoring and testing, virtual instrumentation and distributed measurements, and telecommunication system measurement.

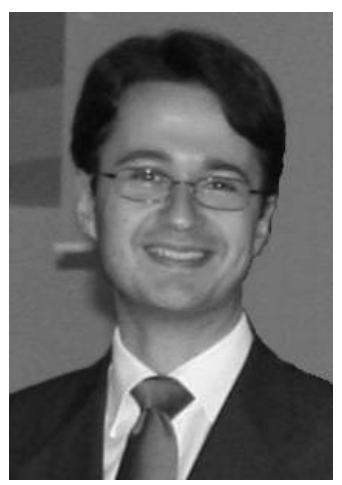

Sergio Rapuano (M'00) received the master's degree (cum laude) in electronic engineering Ph.D. degree, from the University of Salerno (Italy), in computer science, telecommunications and applied electromagnetism. Since 2002 he is with the Faculty of Engineering of the University of Sannio as Assistant Professor of Electric and Electronic Measurement. $\mathrm{He}$ is a member of the IEEE Instrumentation and Measurement Society TC-10 and secretary of the TC23 Working Group on "e-tools for Education in Instrumentation and Measurement". His research interests include digital signal processing for measurement in telecommunications, data converter characterization, distributed measurement systems and virtual laboratories, medical measurement.

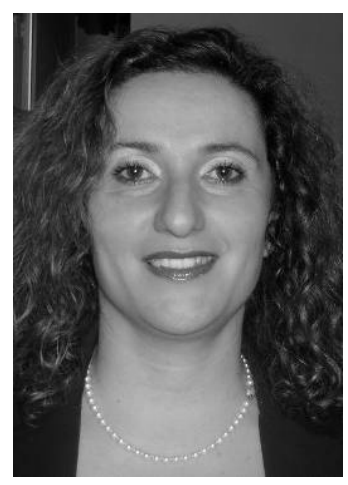

Maria Riccio received, in December 2001, the degree in Computer Engineering at University of Sannio. During 2002 she was a collaborator at Software Engineering Laboratory of the University of Sannio. She was involved in research project regarding development of grid computer infrastructure through Java and XML technologies. Since October 2002 she is Project Manager in Didagroup SpA and she has coordinated projects in the field of online education process e-Learning and to Learning Management System development. Since 2005 she is head of the Research \& Development Center of Didagroup SpA in Benevento. Since May 2007 she is a
PhD student in Computer Engineering. She is a supervisor of Master Thesis regarding e-Learning technologies, remote control of actual instrumentation and didactical processes. She published some scientific papers in international journals and at national and international congresses in the field of remote educational activities.

\section{Francesco Zoino was born in} Benevento,on August 8,1976. In 2004 he achieved the master's degree in Software Engineering from University of Sannio presenting a thesis work titled "A new method for the detection of harmonics in the Power Quality". At the present time he is a $P h D$ Student in Software Engineering and works with the Laboratory of Signal

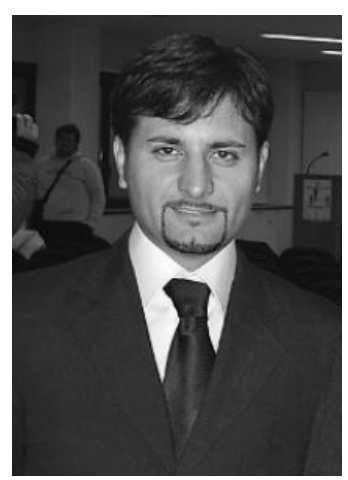
Processing and Measurement Information of the University of Sannio, Benevento. In particular his research work deals with the remote control of measurement instrumentation over the Internet and the medical measurement applications. 